\title{
The Analytical Solution to Zonal Wind-driven Current in a Flat-bottomed Ocean Domain
}

\author{
Zhongfan Zhu
}

\author{
College of Water Sciences, Beijing Normal University, Xinjiekouwai Street 19, 100875 Beijing, China \\ zhuzhongfan1985@gmail.com
}

\begin{abstract}
Study on the zonal wind-driven ocean current is an important subject in the field of physical oceanography. At present, Stommel model and Munk model are being widely used to model this current in a flat-bottomed ocean domain. However, these models are highly idealized and lack the comprehensive consideration of both bottom stress and lateral friction. In this note, the analytical solution to the zonal winddriven current in a flat-bottomed domain is presented in which planetary vorticity, wind stress, bottom stress, and lateral friction are completely included. Comparison with the above two models may suggest that this solution is valid to a certain degree, and this solution may be used to roughly estimate the roles that bottom stress and lateral friction play in the zonal wind-driven ocean current by comparing it with ocean model results or observation data.
\end{abstract}

Keywords-analytical solution;zonal wind;wind-driven ocean current

\section{INTRODUCTION}

Study on the zonal wind-driven ocean current is an important subject in the field of physical oceanography. At present Stommel model [1] and Munk model [2] are two famous models that are being adopted to model the zonal wind-driven current such as the Gulf Stream in the North Atlantic Ocean, the Kuroshio Current in the Pacific Ocean and the Brazil Current in the South Atlantic Ocean [3]. Emphases that these two models stressed are different: Stommel model added the bottom friction term in governing equations of zonal wind-driven ocean current to gain the closed current streamlines and revealed the phenomenon of westward streamline intensification, whereas Munk model considered the importance of lateral friction. It should be pointed out that addition of either bottom stress or lateral friction is random and was performed in order to gain the analytical solution. However, for any engineering case, due to variable water depth and complicated coastline, both bottom stress and lateral friction should play the important role. So it is needed to consider the joint effect of these two frictions. The aim of this note is to present an analytical solution of zonal wind-driven current, without neglecting bottom stress term or lateral friction term.

The layout of this note is as follows: Section 2 summarizes three different analytical solutions of governing equations of zonal wind-driven ocean current: Sverdrup balance, Stommel solution and Munk solution. Based on these, a new analytical solution is derived in Section 3, and subsequently a simple discussion about this solution is given in Section 4.

\section{SUMMARY OF PREVIOUS WORK}

Fig.1 shows the schematic diagram of a wind-driven ocean current in a flat-bottomed domain. Right-handed coordinate system is defined as follows: the origin is in the lower left edge of the domain, the $x$ axis denotes the longitudinal direction, the $y$ axis the lateral direction, and the $z$ axis is positive in the upward direction with the surface level. $H$ is water depth, and $a, b$ are longitudinal length and lateral width of the domain, respectively.

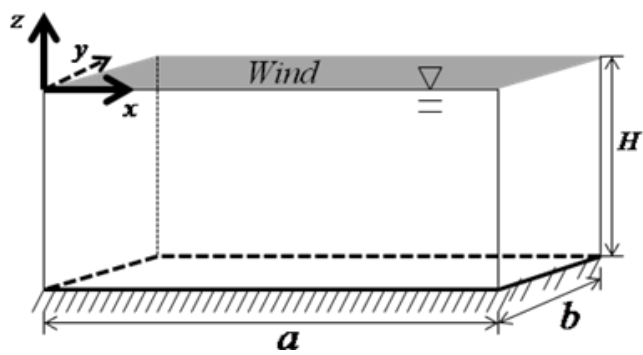

Figure 1. The schematic diagram of wind-driven ocean current in a flatbottomed domain.

The general circulation of wind-driven ocean current can be expressed as follows [3]:

$$
\begin{gathered}
-f v=-\frac{1}{\rho_{0}} \frac{\partial p}{\partial x}+\frac{1}{\rho_{0}}\left(\frac{\partial \tau_{z x}}{\partial z}+\frac{\partial \tau_{x x}}{\partial x}+\frac{\partial \tau_{y x}}{\partial y}\right) \\
f u=-\frac{1}{\rho_{0}} \frac{\partial p}{\partial y}+\frac{1}{\rho_{0}}\left(\frac{\partial \tau_{z y}}{\partial z}+\frac{\partial \tau_{x y}}{\partial x}+\frac{\partial \tau_{y y}}{\partial y}\right) \\
\frac{\partial u}{\partial x}+\frac{\partial v}{\partial y}+\frac{\partial w}{\partial z}=0
\end{gathered}
$$

where $f$ is the Coriolis coefficient, $u, v$ and $w$ the longitudinal, lateral and vertical velocities, respectively, $p, \rho_{0}$ the pressure and density, $\tau$ the stress tensor. If we integrate (1) to (3) for the flat-bottom ocean along the vertical direction and eliminate the pressure term that is included in both (1) and (2), we can have [3]:

$$
\begin{aligned}
& \beta V=\frac{\partial}{\partial x}\left(\frac{\tau_{0 y}}{\rho_{0}}\right)-\frac{\partial}{\partial y}\left(\frac{\tau_{0 x}}{\rho_{0}}\right)+R\left(\frac{\partial V}{\partial x}-\frac{\partial U}{\partial y}\right) \\
& +A\left(\frac{\partial^{3} V}{\partial x^{3}}+\frac{\partial^{3} V}{\partial y^{2} \partial x}-\frac{\partial^{3} U}{\partial x^{2} \partial y}-\frac{\partial^{3} U}{\partial y^{3}}\right)
\end{aligned}
$$

where $\beta=\partial f / \partial y, U, V$ are the vertically-integrated longitudinal and lateral velocities, respectively, $\left(\tau_{0 x}, \tau_{0 y}\right)$ 
are surface wind stress, the bottom stress is $\left(\tau_{-H x}, \tau_{-H y}\right)$ and other vertically-integrated stresses (for example: $\left.T_{X X}=\int_{-H}^{0} \tau_{x x} d z\right)$ are assumed to take the following forms ( $R, A$ are corresponding coefficients):

$\frac{1}{\rho_{0}}\left(\tau_{-H x}, \tau_{-H y}\right)=-R(U, V)$;

$\frac{1}{\rho_{0}}\left(\begin{array}{cc}T_{X X} & T_{Y X} \\ T_{X Y} & T_{Y Y}\end{array}\right)=A\left(\begin{array}{cc}2 \frac{\partial U}{\partial x} & \frac{\partial U}{\partial y}+\frac{\partial V}{\partial x} \\ \frac{\partial U}{\partial y}+\frac{\partial V}{\partial x} & 2 \frac{\partial V}{\partial y}\end{array}\right)$

In (4), the term on the left-hand side represents the planetary vorticity, the first two terms on the right-hand side represents the wind stress curl, the third term bottom stress torque, and the last term lateral friction torque. Introducing a transport streamline function $U=\partial \psi(x, y) / \partial y$, $V=-\partial \psi(x, y) / \partial x$, and assuming coefficients $R^{\prime}=-R$, $A^{\prime}=-A$, this equation can be further reduced to:

$$
\begin{aligned}
& -\beta \frac{\partial \psi(x, y)}{\partial x}=\left[\frac{\partial}{\partial x}\left(\frac{\tau_{0 y}}{\rho_{0}}\right)-\frac{\partial}{\partial y}\left(\frac{\tau_{0 x}}{\rho_{0}}\right)\right]+R^{\prime}\left[\begin{array}{l}
\frac{\partial^{2} \psi(x, y)}{\partial x^{2}}+ \\
\frac{\partial^{2} \psi(x, y)}{\partial y^{2}}
\end{array}\right] \\
& +A^{\prime}\left[\frac{\partial^{4} \psi(x, y)}{\partial x^{4}}+2 \frac{\partial^{4} \psi(x, y)}{\partial y^{2} \partial x^{2}}+\frac{\partial^{4} \psi(x, y)}{\partial y^{4}}\right] .
\end{aligned}
$$

In this equation, the term on the left-handed side denotes planetary vorticity, and among all terms on the right-handed side: the first term represents wind stress curl, the second one bottom stress torque and the last one lateral friction torque.

In this note, we only focus on the zonal wind-driven ocean current. In the sub-tropics, the wind pattern is characterized by the westerly wind in the northern end and the trade wind in the southern end. Assuming that the wind stress is zonal, wind stress can be expressed

$$
\begin{aligned}
\tau_{0 x} & =-\tau_{0} \cos \left(\frac{\pi y}{b}\right), \\
\tau_{0 y} & =0
\end{aligned}
$$

where $\left(\tau_{0 x}, \tau_{0 y}\right)$ denotes the longitudinal and lateral wind stress, respectively, and $\tau_{0}$ is a reference stress, as shown in Fig.2.

In Sverdrup balance, bottom stress torque and lateral friction torque are both ignored and governing equation becomes: $-\beta \partial \psi(x, y) / \partial x=-\partial\left(\tau_{0 x} / \rho_{0}\right) / \partial y$ with a suitable eastern boundary condition $\psi(a, y)=0$. The analytical solution is [3]:

$$
\psi(x, y)=\frac{\tau_{0}}{\rho_{0} \beta}(x-a)\left(\frac{\pi}{b}\right) \sin \left(\frac{\pi y}{b}\right)
$$

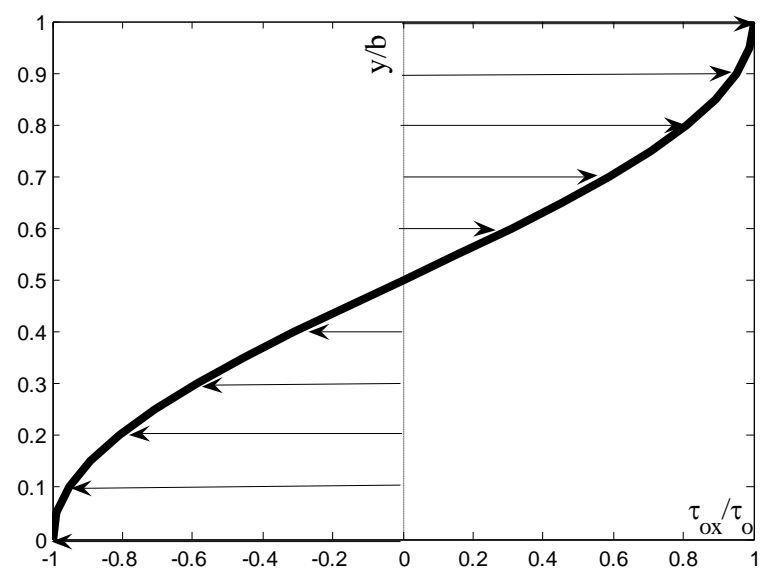

Figure 2. Distribution of zonal wind.

In Munk solution, only lateral friction is retained, bottom stress is neglected and the longitudinal gradient of the lateral friction is further assumed to dominate. Consequently, governing equation becomes:

$$
-\beta \partial \psi(x, y) / \partial x=-\partial\left(\tau_{0 x} / \rho_{0}\right) / \partial y+A^{\prime} \partial^{4} \psi(x, y) / \partial x^{4} .
$$

Subject to boundary conditions $\psi(0, y)=0, \psi(a, y)=0$, $\partial \psi(x, y) /\left.\partial x\right|_{x=0}=0 \quad, \partial \psi(x, y) /\left.\partial x\right|_{x=a}=0$, the analytical solution is gotten, as presented in Mellor [3]:

$$
\psi(x, y)=\frac{\tau_{0}}{\rho_{0} \beta} F(x)\left(\frac{\pi}{b}\right) \sin \left(\frac{\pi y}{b}\right)
$$

where

$$
\begin{aligned}
& F(x)=x-a+a \exp \left(x / 2 \varepsilon_{1} a\right)\left[\begin{array}{l}
\left(1+\varepsilon_{1}\right) \cos \left(\sqrt{3} x / 2 \varepsilon_{1} a\right)- \\
\left(1+3 \varepsilon_{1}\right) / \sqrt{3} \times \sin \left(\sqrt{3} x / 2 \varepsilon_{1} a\right)
\end{array}\right] \\
& -a \varepsilon_{1}\left\{1-\exp \left[(a-x) / \varepsilon_{1} a\right]\right\} .
\end{aligned}
$$

Here, $\varepsilon_{1}^{3}=A^{\prime} /\left(a^{3} \beta\right)$ (for actual engineering case, $\varepsilon_{1}<0$ ). Obviously, when $\varepsilon_{1} \rightarrow 0$, (8) equals to (7).

In Stommel solution, only bottom stress is retained and lateral friction is ignored. By assuming that the $y$ derivative term of the bottom stress can be neglected, governing equation reduces to

$$
-\beta \partial \psi(x, y) / \partial x=-\partial\left(\tau_{0 x} / \rho_{0}\right) / \partial y+R^{\prime} \partial^{2} \psi(x, y) / \partial x^{2}
$$

Adding boundary conditions $\psi(0, y)=0, \psi(a, y)=0$, the solution can be gotten [3]:

$$
\psi(x, y)=\frac{\tau_{0}}{\rho_{0} \beta}\left[a \exp \left(-\frac{x}{\varepsilon_{2} a}\right)+x-a\right]\left(\frac{\pi}{b}\right) \sin \left(\frac{\pi y}{b}\right) .
$$

Here $\varepsilon_{2}=R^{\prime} /(a \beta)$ (for actual engineering case, $\left.\varepsilon_{2}>0\right)$, it is obvious that when $\varepsilon_{2} \rightarrow 0$, (9) is equal to (7). 


\section{NEW ANALYTICAL SOLUTION}

Similar to Munk solution and Stommel solution, we assume that the $y$ derivative term of the bottom stress and the lateral gradient terms of the lateral friction are neglected.Thus governing equation becomes:

$$
-\beta \frac{\partial \psi(x, y)}{\partial x}=-\frac{\partial}{\partial y}\left(\frac{\tau_{0 x}}{\rho_{0}}\right)+R^{\prime} \frac{\partial^{2} \psi(x, y)}{\partial x^{2}}+A^{\prime} \frac{\partial^{4} \psi(x, y)}{\partial x^{4}} .
$$

By observing (8) and (9), we can consider that the analytical solution to (10) has the same form as them and can be expressed to be

$$
\psi(x, y)=\tau_{0} /\left(\rho_{0} \beta\right) \times Q(x)(\pi / b) \sin (\pi y / b) .
$$

Subsitituting this equation into (10) and then using two parameters introduced by Munk solution and Stommel solution, respectively, $\varepsilon_{1}^{3}=A^{\prime} /\left(a^{3} \beta\right), \varepsilon_{2}=R^{\prime} /(a \beta)$ can have

$$
\varepsilon_{1}^{3} a^{3} Q^{(4)}(x)+\varepsilon_{2} a Q^{(2)}(x)+Q^{(1)}(x)-1=0 .
$$

This equation is fourth-order linear non-homogeneous differential equation. So its solution is composed of two parts: a general solution $Q_{g}(x)$ and a special solution $Q_{s}(x):$

$$
\begin{aligned}
& \varepsilon_{1}^{3} a^{3} Q_{g}^{(4)}(x)+\varepsilon_{2} a Q_{g}^{(2)}(x)+Q_{g}^{(1)}(x)=0 \\
& \varepsilon_{1}^{3} a^{3} Q_{s}^{(4)}(x)+\varepsilon_{2} a Q_{s}^{(2)}(x)+Q_{s}^{(1)}(x)-1=0 .
\end{aligned}
$$

By observing (8) and (9) again, we can define $Q_{s}(x)=x-a$. Obviously, $Q_{s}(x)$ satisfy (13). Assuming $Q_{g}(x)=\exp (\lambda x)$, we can know that $\lambda$ should be the root of the following characteristic equation:

$\varepsilon_{1}^{3} a^{3} \lambda^{4}+\varepsilon_{2} a \lambda^{2}+\lambda=0$. Its four roots are: $\lambda_{1}=0$, $\lambda_{2}=-2 k_{1}, \lambda_{3}=k_{1}+k_{2} * i, \lambda_{4}=k_{1}-k_{2} * i$,

where

$$
k_{1}=\frac{\left.\left.\sqrt[3]{\frac{1}{2}\left[27 \varepsilon_{1}^{6} a^{6}+\sqrt{\left(27 \varepsilon_{1}^{6} a^{6}\right)^{2}-4\left(-3 \varepsilon_{1}^{3} \varepsilon_{2} a^{4}\right)^{3}}\right.}\right]+3 \sqrt{\frac{1}{2}\left[27 \varepsilon_{1}^{6} a^{6}-\sqrt{\left(27 \varepsilon_{1}^{6} a^{6}\right)^{2}-4\left(-3 \varepsilon_{1}^{3} \varepsilon_{2} a^{4}\right)^{3}}\right.}\right]}{6\left(\varepsilon_{1} a\right)^{3}}
$$

$k_{2}=\sqrt{3} \frac{\left.\sqrt[3]{\frac{1}{2}\left[27 \varepsilon_{1}^{6} a^{6}+\sqrt{\left(27 \varepsilon_{1}^{6} a^{6}\right)^{2}-4\left(-3 \varepsilon_{1}^{3} \varepsilon_{2} a^{4}\right)^{3}}\right]}-3 \sqrt{\frac{1}{2}\left[27 \varepsilon_{1}^{6} a^{6}-\sqrt{\left(27 \varepsilon_{1}^{6} a^{6}\right)^{2}-4\left(-3 \varepsilon_{1}^{3} \varepsilon_{2} a^{4}\right)^{3}}\right.}\right]}{6\left(\varepsilon_{1} a\right)^{3}}$

As a result, the stream function $\psi(x, y)$ has this form

$\psi(x, y)=\frac{\tau_{0}}{\rho_{0} \beta}\left\{\begin{array}{l}C_{1}+C_{2} \exp \left(-2 k_{1} x\right)+\exp \left(k_{1} x\right) \times \\ {\left[C_{3} \cos \left(k_{2} x\right)+C_{4} \sin \left(k_{2} x\right)\right]+x-a}\end{array}\right\}\left(\frac{\pi}{b}\right) \sin \left(\frac{\pi y}{b}\right)$

where $C_{1}, C_{2}, C_{3}, C_{4}$ are real values. Considering boundary conditions: $\psi(0, y)=0, \psi(a, y)=0, \partial \psi(x, y) /\left.\partial x\right|_{x=0}=0$, $\partial \psi(x, y) /\left.\partial x\right|_{x=a}=0$, we can get:

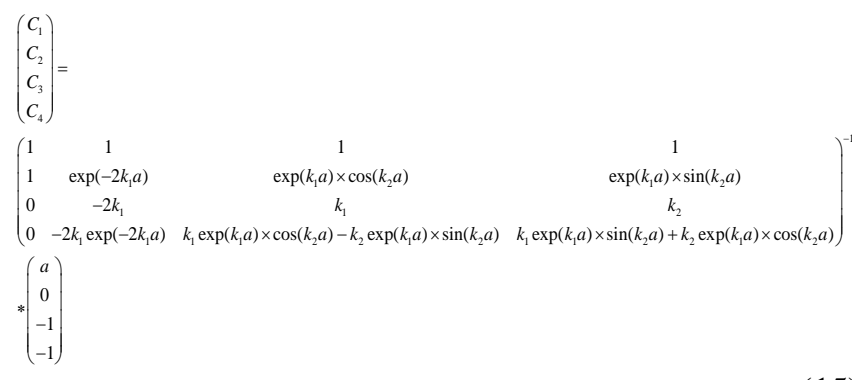

Moreover, the vertically-integrated lateral velocity $V$ becomes:

$V=-\partial \psi(x, y) / \partial x=\tau_{0} /\left(\rho_{0} \beta\right) \times Q^{(1)}(x)(\pi / b) \sin (\pi y / b)$,

where

$$
\begin{aligned}
& Q^{(1)}(x)=-2 k_{1} C_{2} \exp \left(-2 k_{1} x\right)+k_{1} \exp \left(k_{1} x\right) \times\left[C_{3} \cos \left(k_{2} x\right)+C_{4} \sin \left(k_{2} x\right)\right] \\
& +\exp \left(k_{1} x\right) \times\left[-C_{3} k_{2} \sin \left(k_{2} x\right)+C_{4} k_{2} \cos \left(k_{2} x\right)\right]+1
\end{aligned}
$$

\section{DISCUSSION}

In order to present this analytical solution, we adopt a simple engineering example introduced in Mellor [3] and in this example all of the parameter values are: $\varepsilon_{1}=-0.05$, $\varepsilon_{2}=0.025, \tau_{0}=1 \mathrm{dyne} / \mathrm{cm}^{2}, \quad \beta=2 \times 10^{-8} \mathrm{~km}^{-1} \mathrm{~s}^{-1}$, $a=6000 \mathrm{~km}, b=4000 \mathrm{~km}$. We conveniently adopt the vertically-integrated lateral velocity $V$ at $y=b / 2$ to compare this analytical solution and Stommel and Munk solutions, as shown in Fig.3. Stommel solution is infinite on the western boundary $(x=0)$ whereas the analytical solution is null. The combined inclusion of bottom stress and lateral friction allow for addition of non-slip condition. Distribution of the analytical solution across the domain is very similar to Munk solution, and in particular they are completely same in the region far from the western boundary $(x / a \geq 0.45)$. The difference between them is that the peak of the analytical solution is larger than Munk solution and the trough is smaller than Munk solution, which is due to addition of bottom stress. These may suggest that the analytical solution is valid to a certain degree.

At present, some kinds of ocean models have been developed and data sets of observation of ocean currents are available. It seems to be possible to roughly understand roles that bottom stress and lateral friction play in the zonal wind-driven ocean current by comparing the analytical solution drived in this note with ocean model results or observation data sets.

\section{REFERENCES}

[1] H. Stommel, "The westward intensification of wind-driven ocean currents,". Transactions, American Geophysical Union, vol. 29(2), 1948, pp.202-206.

[2] W.H. Munk, "On the wind-driven ocean circulation," Journal of meteorology, vol. 7(2), 1950, pp. 78-93.

[3] G.L. Mellor, Introduction to Physical Oceanography, AIP Press, New York,1996, pp. 84-102. 


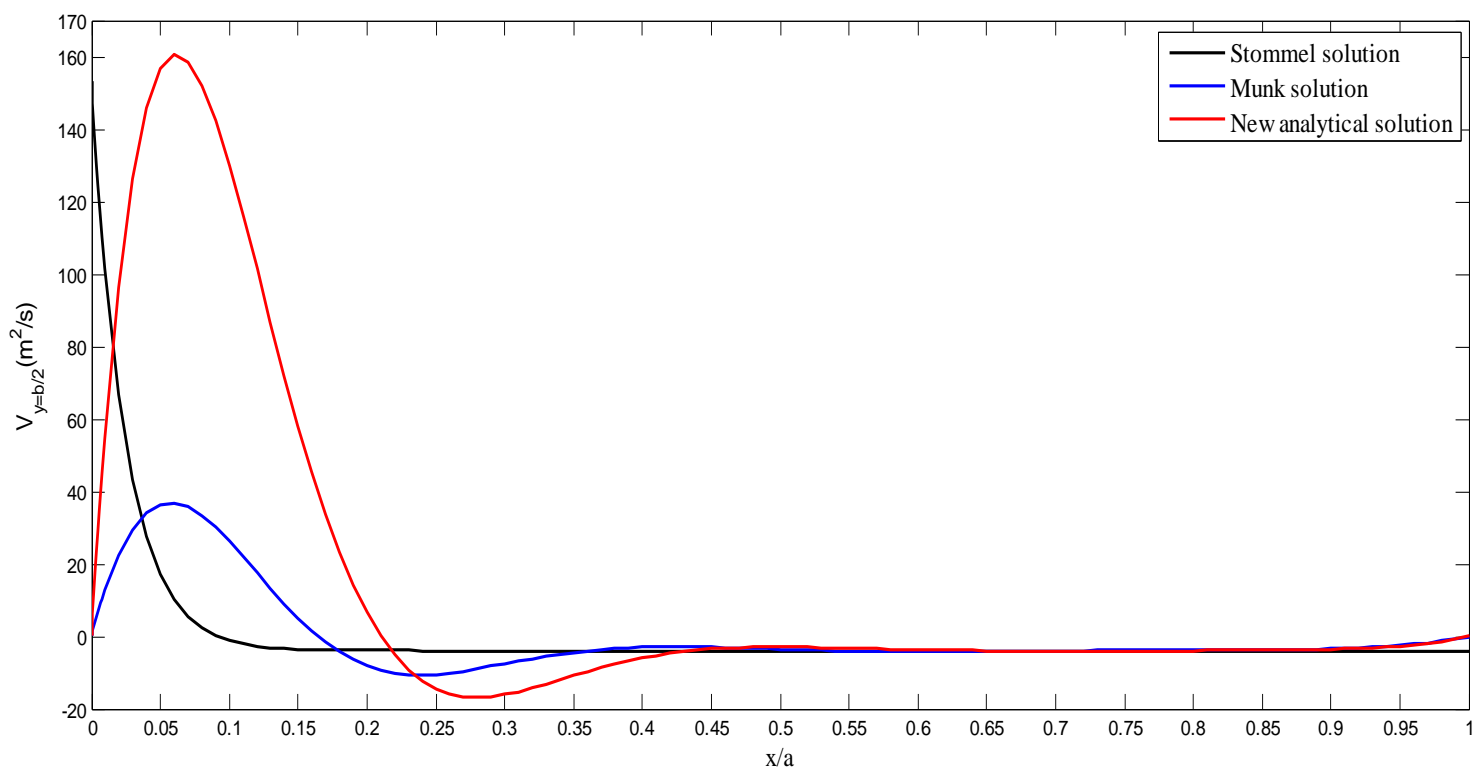

Figure 3. The vertically-integrated lateral velocity $V$ at $y=b / 2$ over the domain for three different solutions. The analytical solution corresponds to $\varepsilon_{1}=-0.05, \varepsilon_{2}=0.025$, and Stommel and Munk solutions correspond to $\varepsilon_{2}=0.025, \varepsilon_{1}=-0.05$, respectively. The unit of $V$ is $\mathrm{m}^{2} / \mathrm{s}$. 